\title{
An Evolutionary Approach to Underwater Sensor Deployment
}

\author{
Erik F. Golen ${ }^{*}$ \\ Laboratory for Wireless Networking and Security \\ B. Thomas Golisano College of Computing and Information Sciences \\ Rochester Institute of Technology \\ Bo Yuan \\ Networking, Security, and Systems Administration Department \\ B. Thomas Golisano College of Computing and Information Sciences \\ Rochester Institute of Technology \\ E-mail:bo.yuan@rit.edu \\ www.nssa.rit.edu \\ Nirmala Shenoy \\ Networking, Security, and Systems Administration Department \\ B. Thomas Golisano College of Computing and Information Sciences \\ Rochester Institute of Technology \\ E-mail:nxsvks@rit.edu \\ www.nssa.rit.edu/ ns \\ Received: 18/02/09 \\ Accepted: 06/07/09
}

\begin{abstract}
Underwater sensor deployment for military surveillance is a significant challenge due to the inherent difficulties posed by the underwater channel in terms of sensing and communications between sensors, as well as the exorbitant cost of the sensors. As a result, these sensors must be deployed as efficiently as possible. The proposed Underwater Sensor Deployment Evolutionary Algorithm considers six important factors that have not yet been simultaneously considered due to the ensuing complexity of the problem. Two principle studies are presented in this work, a benchmarking study that shows the effectiveness of the algorithm and a simulation study that outlines the inevitable effect of communications range on the sensing capabilities on an underwater sensor field.
\end{abstract}

Keywords: Underwater sensor networks, sensor deployment strategies, range dependence, evolutionary algorithms.

* Rochester Institute of Technology, 1 Lomb Memorial Drive, Rochester, NY 14623, United States of America, efg5752@rit.edu, www.rit.edu. 


\section{Introduction}

Underwater sensor networks are often used to monitor a general area of the ocean, a port or military installation, or to detect underwater vehicles near a high value unit at sea, such as a fuel ship or aircraft carrier. Any suspicious activity is reported by the sensors via a floating surface station to a central location, for example, a surface ship or a command station on land. Based on the reports, decisions are made on how to proceed against the threat [1]. The focus of this study is to investigate deployment strategies for underwater acoustic sensors, using an evolutionary algorithmic approach.

An evolutionary approach (EA) is well suited to optimizing non-linear objective functions, while applying several constraints, which are a necessity for underwater sensor deployments. An EA is a powerful offline search tool, which can be used to efficiently search a large solution space in a parametric manner, allowing field designers to perform a wide variety of studies before a sensor field is deployed. The EA searches will ultimately provide field designers with heuristics for determining the composition of an efficient underwater sensor deployment. Lastly, the inherent flexibility of an EA allows for the relaxation of assumptions at the discretion of the field designer and the inclusion of several relevant factors for underwater sensor deployments.

Deploying underwater acoustic sensors poses several challenges to field designers. Two major challenges are addressed in this article. First, determining the impacts of the underwater channel on the sensors in terms of sensing and communication capabilities is difficult. Secondly, the number of sensors available to field designers is limited by the high costs of such sensors and the associated high costs for deployment of these sensors [2].

Looking at the first challenge, the underwater channel introduces signal attenuation due to geometric spreading and absorption by the medium. Moreover, multipath and man-made and ambient noise causes significant interference [1-3]. As a result, sensing and communications ranges, bandwidth, and data rates are limited [2]. Also, extremely high propagation delays occur since the propagation speed of a signal in water is 5 orders of magnitude slower than in the terrestrial channel [2]. Such high delays will severely limit channel utilization and throughput. It must also be noted that the speed of sound in water, and therefore delays, is range dependent, meaning that it varies by geographic location [4,5]. For these reasons, adopting models and studies conducted for transmission over terrestrial channels is not a viable option.

Additionally, sound speed is dependent upon water temperature, depth, salinity, and time of year [4]. Permanent losses of connectivity between sensors may occur due to the existence of shadow zones that cannot be accounted for a priori. Bubble clouds, caused by crashing waves, can cause intermittent disruptions in connectivity [2]. As this study focuses on acoustic sensors, both underwater sensing and communication are done acoustically. Thus, both are affected by the problems of the underwater channel [1].

To address the above challenges in this article we focus on six main factors that we believe to be of paramount importance for underwater military surveillance networks, including

- $\quad$ sensing range

- communications range

- $\quad$ sensor and deployment costs

- link redundancy

- $\quad$ range dependence of the environment

- $\quad$ probabilistic visitation

Many of the more popular sensor deployment strategies, such as the ones noted in [3,6-9], tend to focus mainly on the sensing range of a sensor and the sensor field. We feel, however, that it is important to consider both the sensing range and the communications range. If sensors are deployed such that they are not within communications range of each other and/or a data sink, it becomes impossible to aggregate the sensed data.

Communications range: As our study takes into account the communications range of a sensor, it was important to decide on a topology that would help aggregate the data. Mesh and cluster topologies are two of the more popular topologies used in ad hoc networks and hence are considered in this article. These two topologies are discussed in more detail in Section 3.1 and, as will be discussed, each has a unique effect on the sensing capabilities of a sensor field.

Sensor and deployment costs: Cost is a major factor in underwater sensor networks. Hence, it is logical that the limited number of sensors available to the field 
designer should be deterministically deployed in an efficient manner, rather than randomly deployed [3]. Our studies assume a limited number of sensors, which are to be deployed with the goal of maximizing field sensing capabilities, while meeting the connectivity requirements of the chosen topology.

Link redundancy: The characteristics of the underwater channel can result in intermittent and even permanent losses on connectivity. This requires multiple communication paths, or link redundancy. Link redundancy is especially important if the sensor field is to be deployed in a particularly harsh environment.

Range dependence denotes the non-negligible effect sensor location has on sensing and communications ranges. This is an artifact of the variability of sound speed and bathymetric phenomena, such as changes in depth over a region, which in turn varies the transmission loss of sound over distance [4]. Such variabilities suggest that an underwater sensor deployment strategy should utilize environmental data from historical databases, or in situ, to calculate realistic values for sensing and communications ranges throughout an area of interest.

Probabilistic visitation is the likelihood that an adversary will visit a sector, which is defined as a particular region of unique acoustic characteristics. An area of interest could be comprised of several such sectors [5], as a result of variabilities in the environment. If it can be probabilistically determined that an adversary is more likely to visit one sector over another, more sensors should be allocated to that sector. Probabilistic visitation can be computed using game theory [5]. This knowledge provides a field designer with an allocation scheme suited to each sector

Link redundancy, range dependence, and probabilistic visitation are important factors that have not been considered in any sensor deployment work to date to the best of our knowledge. We feel that not considering all six of the above factors, and the correlated effects, in an underwater sensor deployment strategy could result in an ineffective sensor deployment.

The proposed Underwater Sensor Deployment Evolutionary Algorithm (USDEA) considers all of these factors.

Two main contributions of this work, in addition to designing the USDEA, are 1) a standard benchmarking of the algorithm and 2) a study of the effect of communications range on the sensing capabilities of sensor fields using mesh and cluster topologies. The first study will show the effectiveness of the algorithm as compared with hill climbing and random search. The second study shows the effect the communications range and each topology has on the sensing capabilities of a sensor field.

The remainder of this paper is organized as follows. A brief literature review is given in Section 2, followed by a presentation of the topologies used in the USDEA in Section 3. A detailed discussion of the USDEA is provided in Section 4. Section 5 presents the benchmarking study, while Section 6 shows the effect of communications range on the sensing capabilities of both mesh and cluster fields. Further analysis of these results is presented in Section 7 and conclusions are drawn in Section 8.

\section{Related Works}

Numerous works exist in the literature for sensor deployment in terrestrial sensor networks [6-9], while relatively few can be noted for underwater sensor networks [3].

In [6], Voronoi diagrams and Delaunay Triangulation are used to determine the worst and bestcase coverage, respectively, of a field of randomly deployed sensors. These two computational geometric constructs are used to find optimal locations to deploy additional sensors so that the worst or best-case field coverage can be improved. However, it is assumed that a sensor's location has no effect on its sensing capabilities. By not considering the range dependent effects of the environment, a sensor field is unlikely to perform in practice as it does in principle.

A similar approach to improving the worst-case coverage of a random sensor deployment is shown in [7], where an area is divided into grid cells. Additional sensors can only be placed at the intersection point of the grid cells. Such an approach restricts the number of possible sensor locations to the granularity of the grid cells, which may result in suboptimal deployments. Since the number of available underwater sensors is limited due to cost constraints, random deployments may not be cost efficient.

In [8], a Virtual Forces algorithm is proposed, where sensors repel each other if they are too close and attract each other if they are too far apart. Sensors are initially deployed at random, and based upon the repulsive and 
attractive forces, reposition themselves. Sensing coverage is measured in terms of how well a sensor field covers a set of distinct points, rather than the coverage of the general area. In this article also sensing is assumed to be range independent and that underwater sensors are mobile, which could increase the cost factor of the resulting sensor field.

A 2D underwater sensor deployment strategy that arranges sensors in a triangular pattern to minimize sensing coverage overlap is proposed in [3]. Its goal is to completely cover a region in terms of sensing, using a minimum number of sensors. However, the deployment strategy assumes the availability of hundreds, and even thousands, of sensors, which may not be monetarily feasible.

It is worth noting that none of the previously mentioned deployment strategies consider communications ranges. This is problematic, as in the underwater case, communications ranges are typically less than sensing ranges, and thus are a limiting factor to the achievable sensing coverage of a field [1].

The first evolutionary approach to deploying sensors can be found in [9], where a genetic algorithm (GA) is used to optimize two competing objectives, sensing coverage and energy consumption. Unlike the previous works, both sensing and communications ranges are considered in this work. A high-energy communications node is assumed to be in the center of the network, which effectively limits the manner in which the sensors can be deployed. When the ratio of the sensing to the communications range is less than 0.5 , the sensors are deployed in a beehive configuration. However, when the ratio is greater than 0.5 , the sensors are deployed in a hub and spoke configuration. [9] further assumed range independence in the environment.

As can be noted, none of the sensor deployment strategies have taken into account all six factors that were highlighted earlier. Most of these deployment strategies, except for the last one, did not consider the impact of communications range on the achievable sensing coverage of a field. Hence, a performance comparison between the proposed USDEA and the above cited works would be unfair.

\section{Sensor Field Types}

Two predominant topologies used in ad hoc networks are considered in this work, the mesh topology and the cluster-based topology. Each has its unique strengths and weaknesses. Mesh fields provide redundant communication paths through the network to enhance connectivity. However, this could reduce the sensing coverage of the field, as the sensors have to be placed closer together in order to achieve the desired level of connectivity. On the other hand, cluster fields, with one hop clusters formed around dedicated cluster heads, will result in single points of failure. Sensing coverage can be improved by increasing the number of clusters. For the purposes of communications, these topological constraints are imposed on the USDEA. This section provides the details on how the mesh and cluster based topologies are created and evaluated by the USDEA.

\subsection{Mesh Fields}

A mesh field is deployed such that each sensor is within the communications range of some number of other sensors, to provide multiple communication paths through the network. Recall that multiple communication paths are necessary for overcoming problems such as losses in connectivity caused by shadow zones and bubble clouds. Such losses in connectivity can result in delays in transmitting time critical sensed data (e.g. when an adversary is within the sensing range of a sensor) to a floating surface station.

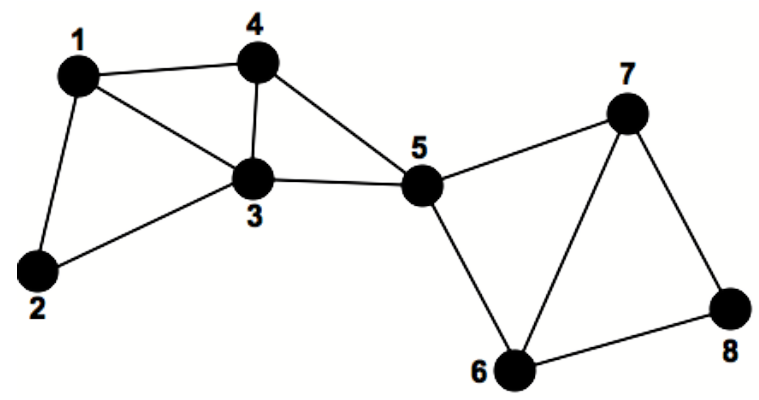

Fig.1. Example Mesh Field

In a mesh field, link redundancy is measured by determining the minimum cut set size, which is the number of links that must be removed in order to segment a network [10]. In Fig. 1, for example, if a shadow zone removed the link between Nodes 4 and 5 and Nodes 3 and 5, the network would be segmented. As a result, information about a target detected by Node 7 could not be relayed to Node 1, which would be highly problematic, assuming Node 1 is the node closest to a floating surface station. Stoerr's algorithm [10] 
applies graph theory to determine the minimum cut set size of a mesh field.

A logical inference is that as the minimum cut set size of a mesh field is increased, an appreciable decrease in the sensing coverage by the field will result.

\subsection{Cluster Fields}

Cost is a major consideration in cluster fields, as there are not only a limited number of sensors, but also, a limited number of dedicated high-energy cluster heads that can communicate directly with a surface station. These cluster heads act as sinks for the one-hop cluster client sensors and transmit the aggregated data to the surface stations. One hop clusters, as opposed to multi hop, minimize latency in transmitting detection data to the surface station.

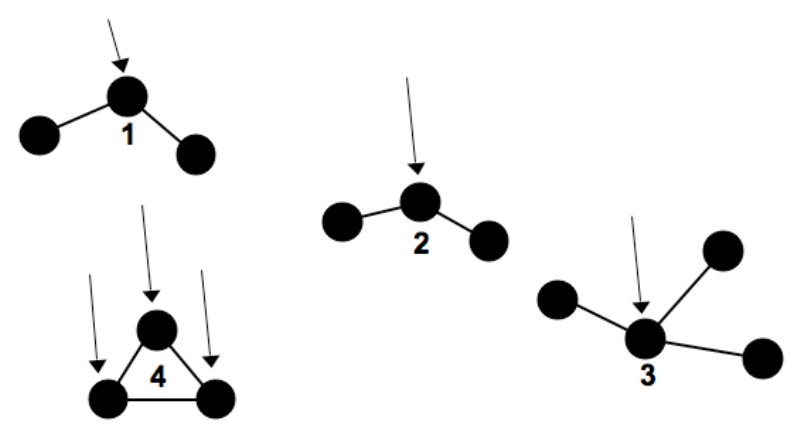

Fig.2. Example Cluster Field

Fig. 2 shows a cluster field of 4 clusters. The nodes with arrows pointing to them are eligible to be cluster heads, based upon their location. The following algorithm is used to determine such eligible cluster heads prior to deployment.

A node is a cluster head if it has the highest degree locally. That is, it has the most nodes within its communications range compared to all of its neighboring nodes. All nodes one hop from a cluster head are therefore not eligible to be a cluster head. Note that in Cluster 4, all of the nodes have a degree of 2 , thus any of them can be a cluster head.

As opposed to mesh fields, where the sensors create a single region of sensing coverage, cluster fields will have multiple clusters scattered throughout a sector, with their own unique sensing coverage regions.

\section{The Underwater Sensor Deployment Evolutionary Algorithm}

This section describes how a sensor field is represented in the USDEA and discusses each of the steps used in the algorithm.

\subsection{Sensor Field Representation}

Sensor fields are represented in the USDEA as an unordered set of $n$ Cartesian sensor locations, as shown below in Eq. (1).

$$
F=\left\{\left(x_{s_{1}}, y_{s_{1}}\right),\left(x_{s_{2}}, y_{s_{2}}\right), \ldots\left(x_{s_{n}}, y_{s_{n}}\right)\right\}
$$

$F$ is a chromosome and each 2D Cartesian coordinate, which represents a sensor location, is a gene. Note that each sensor is assumed to be at the same depth for the purposes of this work, as the metric under consideration is the sensing capabilities of the sensor field. As the sensors under consideration are acoustic, a 2D deployment for sensing purposes is sufficient since the entire water column is insonified by an adversary [4]. In cases where a surface duct is present, the sensors should be deployed below the duct, due to the likelihood that an adversary will spend a large portion of its patrol time well below periscope and duct depth. 3D sensor deployment is planned as a future study to reduce the effects of multipath on communications by sending transmissions in the vertical, rather than horizontal, channel as often as possible. However, that is beyond the scope of this work.

\subsection{Underwater Sensor Deployment Evolutionary Algorithm Steps}

The steps in the execution of the USDEA are listed below.

Step 1: Initial Population Construction

Step 2: Field Evaluation and Fitness Calculation

Step 3: Order Fields by Fitness

Step 4: Remove Least Fit Fields from Population

Step 5: Crossover

Step 6: Mutation

Return to Step 2 until Max Generations Reached

In Step 1, the algorithm begins by creating an initial population of mesh or cluster fields within a single 
sector. An area can be divided into sectors using a learning algorithm, such as a self-organizing map (SOM), that can analyze historical or in-situ acoustic data for that area during a particular time of year that the sensor field will be deployed. The sectors are obtained so that the acoustics within are close to uniform. Each sensor field is independently optimized per sector, with a fixed number of sensors being allocated to each sector prior to the start of the algorithm. This allocation may be determined using the game theoretic approach to underwater sensor allocation described in [5].

The fitness of each sensor field is determined and then the fields are sorted by fitness, with the top performing fields surviving to the next generation. Lastly, evolutionary operators, crossover and mutation, are performed on the surviving population members before the next generation begins.

Each of the steps in the USDEA is discussed in more detail below, except for Steps 3 and 4, as they are self-explanatory.

\section{Step 1: Initial Population Construction}

In Step 1 of the USDEA, an initial population of mesh or cluster fields is constructed within a sector using the algorithm explained below.

Assume $R_{\text {comm }}$ is the communications range of a sensor within the sector. For both types of fields, the first sensor is placed at a random location. With mesh fields, the next sensor is placed at a random distance $R_{d}$ from the first sensor at a random angle $\theta$ where,

$$
\begin{aligned}
R_{d} & =\left[0, R_{\text {comm }}\right] \\
0 & \leq \theta<360
\end{aligned}
$$

Each subsequent sensor location is derived based upon the location of any of the previously placed sensors, chosen at random.

For cluster fields, the same method is followed, except that a sensor can be placed based upon the location of a currently existing sensor, or can be deployed to a random location, which will start a new cluster.

\section{Step 2: Field Evaluation and Fitness Calculation}

Each new sensor field constructed by the USDEA must undergo an evaluation step. First, an undirected connectivity graph of the sensor field is generated. Each graph is evaluated, as shown below, to determine its connectivity properties, which are compared with a threshold value defined for the sector. This value is defined by the field designer and depends on the harshness of the environment.

The threshold value is either a minimum cut set size (MCSS) in the case of a mesh field or a maximum number of clusters (MNC) in the case of a cluster field. If the threshold value is not met, the field is given a fitness of 0 in order to prevent it from surviving to the next generation. For example, if an MCSS of 4 is required and the field only has an MCSS of 3, that field is given a fitness of 0 , so that it is unlikely to survive to the next generation.

Using the undirected connectivity graph for each field, the MCSS or MNC is calculated, as described in Section 3.1.

If the field meets the threshold value, the sector is divided into 1 square kiloyard $\left(1 \mathrm{kyd}^{2}=0.836 \mathrm{~km}^{2}\right)$ grid cells and the signal excess of each cell is calculated with respect to each sensor in the field, using Urick's Passive Sonar Equation [4].

$$
S E_{i, j_{S_{k}}}=S L-T L-N L-D T+D I
$$

where

- $\quad S E=$ Signal excess at a grid cell at location $(i, j)$ with respect to the location of sensor $k$.

- $S L=$ Source level is the intensity of the sound emitted by an underwater adversary.

- $T L=$ Transmission loss is signal loss with distance as a result of propagation through the water and is representative of a sector's acoustic characteristics. This loss is a result of both frequency-based absorption by the medium and geometric spreading [4].

- $D I=$ Directivity index is a gain based upon how well the sensor can determine the direction of the sound.

- $D T=$ Detection threshold is the signal-to-noise ratio required by the sensor to differentiate an adversary from the medium. 
- $\quad N L=$ Noise Level is indicative of the water depth, shipping level, and wind speed in a sector [4].

It should be noted that all values in Eq. (4) are in $d B$ re $\mu \mathrm{Pa}$ (read 1 micro Pascal of pressure). This $1 \mu \mathrm{Pa}$ reference value is equivalent to $0.67 \times 10^{-22} \mathrm{Watts} / \mathrm{cm}^{2}$ [4]. A signal excess of 0 implies that the instantaneous, or stationary, probability of detecting an adversary in that grid cell is 0.50 . As the signal excess becomes more positive, the stationary probability of detection (PDS) increases [4]. The probability is stationary as it is an average detection probability by the sensors with respect to a particular grid cell and is not a temporal measure of detection.

$$
S E_{i, j}=\max \left(S E_{i, j_{s_{1}}}, S E_{i, j_{S_{2}}}, \ldots, S E_{i, j_{s_{n}}}\right)
$$

In Eq. (5), the signal excess of a grid cell at location $(i, j)$ is the maximum signal excess of all the sensors, $s_{k}$, in the field, relative to that grid cell.

$$
\begin{gathered}
P D S_{i, j}=\left\{\begin{array}{l}
S E_{i, j} \geq 0, \frac{1}{2} C^{-16} \\
S E_{i, j}<0,1-\frac{1}{2} C^{-16}
\end{array}\right. \\
C=\sum_{i=0}^{6} A_{i} P^{i} \\
P=\left|\frac{S E_{i, j}}{\sqrt{2} * S D}\right|
\end{gathered}
$$

Eqs. (6-8), presented in [4], convert the signal excess of a grid cell to a PDS. Eqs. (6-7) are a 6-degree polynomial curve fitting that allows for a conversion from signal excess to PDS, where $A=[1,0.0705$, $0.04223,0.00927,0.000152,0.000277,0.00000431]$. In Eq. (8), $S D$ is the standard deviation of the normal distribution, typically $6 \mathrm{~dB}$, for passive sonar [4].

Once the signal excess for each grid cell has been converted to a PDS, the overall fitness of the sensor field is calculated using Eq. (9).

$$
\text { Fitness }=\frac{\sum_{i=1}^{\text {length }} \sum_{j=1}^{\text {width }} P D S_{i, j}}{\text { length } * \text { width }}
$$

The fitness of the field is the average PDS across all grid cells in the sector. Parameters length and width denote how many grid cells in the $\mathrm{x}$ and $\mathrm{y}$ direction, respectively, exist within the sector. This fitness function provides little analytical meaning, but is useful for predicting simulated sensor field performance. As the fitness function value increases, the simulated detection capabilities of the sensor field also increases.

\section{Step 5: Crossover}

Crossover, Step 5 of the algorithm, is executed between a given percentage of the fittest fields, starting with the

\begin{tabular}{|c|c|c|c|c|c|c|}
\hline \multicolumn{7}{|c|}{ Parent $i$} \\
\hline $\begin{array}{c}\text { (i1) } \\
x_{i 1}, y_{i 1}\end{array}$ & $\begin{array}{c}\mathrm{i} 2 \\
x_{\mathrm{i} 2}, y_{\mathrm{i} 2} \\
\end{array}$ & $\begin{array}{c}\mathrm{i} 3 \\
x_{\mathrm{i} 3}, y_{\mathrm{i} 3} \\
\end{array}$ & $\begin{array}{c}i 4 \\
x_{i 4}, y_{i 4} \\
\end{array}$ & $\begin{array}{r}\text { (i5) } \\
x_{i 5}, y_{i 5}\end{array}$ & $\begin{array}{c}\text { (i6) } \\
x_{i 6}, y_{i 6}\end{array}$ & $\begin{array}{c}i 7 \\
x_{i 7}, y_{i 7} \\
\end{array}$ \\
\hline \multicolumn{7}{|c|}{ Parent $j$} \\
\hline$\underset{x_{j 1}, y_{j 1}}{\text { (j1) }}$ & $\begin{array}{c}j 2 \\
x_{j 2}, y_{j 2}\end{array}$ & $\begin{array}{c}j 3 \\
x_{j 3}, y_{j 3}\end{array}$ & $\begin{array}{c}j 4 \\
x_{j 4}, y_{j 4}\end{array}$ & $\mathrm{x}_{\mathrm{j} 5}, \mathrm{y}_{\mathrm{j} 5}$ & $x_{j 6}, y_{j 6}$ & $\begin{array}{c}j 7 \\
x_{j 7}, y_{j 7}\end{array}$ \\
\hline \multicolumn{7}{|c|}{ Child 1} \\
\hline $\begin{array}{c}j 1 \\
x_{j 1}, y_{j 1}\end{array}$ & $\begin{array}{c}\mathrm{i} 2 \\
\mathrm{x}_{\mathrm{i} 2}, \mathrm{y}_{\mathrm{i} 2}\end{array}$ & $\begin{array}{c}\mathrm{i} 3 \\
\mathrm{x}_{\mathrm{i} 3}, \mathrm{y}_{\mathrm{i} 3}\end{array}$ & $\begin{array}{c}i 4 \\
x_{i 4}, y_{i 4} \\
\end{array}$ & $\begin{array}{c}j 5 \\
x_{j 5}, y_{j 5}\end{array}$ & $\begin{array}{c}j 6 \\
x_{j 6}, y_{j 6}\end{array}$ & $\begin{array}{c}i 7 \\
x_{i 7}, y_{i 7}\end{array}$ \\
\hline \multicolumn{7}{|c|}{ Child 2} \\
\hline $\begin{array}{c}i 1 \\
x_{i 1}, y_{i 1}\end{array}$ & $\begin{array}{c}j 2 \\
x_{j 2}, y_{j 2}\end{array}$ & $\begin{array}{c}j 3 \\
x_{j 3}, y_{j 3}\end{array}$ & $\begin{array}{c}j 4 \\
x_{j 4}, y_{j 4}\end{array}$ & $\begin{array}{c}\text { i5 } \\
\mathrm{x}_{\mathrm{i5}}, \mathrm{y}_{\mathrm{i} 5}\end{array}$ & $\begin{array}{c}i 6 \\
x_{i 6}, y_{i 6}\end{array}$ & $\begin{array}{c}j 7 \\
x_{j 7}, y_{j 7}\end{array}$ \\
\hline
\end{tabular}
highest, and a randomly chosen partner. Once a partner is chosen, a random number of genes (sensor locations), chosen at random, between 1 and $n / 2$ (rounded down) are exchanged, resulting in two children.

Fig. 3. An Example of Crossover

The crossover operator is demonstrated in Fig. 3 between Parents $i$ and $j$. Each chromosome is of size 7, thus between 1 and 3 genes can be swapped in the crossover. In this example, 3 sensor locations are swapped. As can be seen, sensor locations 1, 5, and 6 from Parent $j$ are merged with the remaining sensor locations from Parent $i$, resulting in Child 1. Similarly, sensor locations 1, 5, and 6 from Parent $i$ are merged with the remaining sensor locations from Parent $j$, resulting in Child 2. Each child's fitness is then evaluated, as defined in Step 2 of the USDEA. 


\section{Step 6: Mutation}

Mutation is attempted per gene, with a mutation probability $\left(\mu_{m}\right)$ specified by the field designer, to prevent preliminary convergence of the USDEA and provide variation in the population.

In the case of mutation, a new random sensor location is chosen for the gene and the altered chromosome becomes a new population member.

Parent $i$

\begin{tabular}{|c|c|c|c|c|c|c|}
\hline $\begin{array}{c}i 1 \\
x_{i 1}, y_{i 1}\end{array}$ & $\begin{array}{c}\mathrm{i} 2 \\
\mathrm{x}_{\mathrm{i} 2}, \mathrm{y}_{\mathrm{i} 2}\end{array}$ & $\begin{array}{c}\text { i3 } \\
x_{i 3}, y_{i 3}\end{array}$ & (i4) & $\begin{array}{c}i 5 \\
x_{i 5}, y_{i 5}\end{array}$ & $\begin{array}{c}i 6 \\
x_{i 6}, y_{i 6}\end{array}$ & $\begin{array}{c}i 7 \\
x_{i 7}, y_{i 7}\end{array}$ \\
\hline \multicolumn{7}{|c|}{ Member 1} \\
\hline $\begin{array}{c}\mathrm{i1} \\
\mathrm{x}_{\mathrm{i} 1}, \mathrm{y}_{\mathrm{i} 1}\end{array}$ & $\begin{array}{c}\mathrm{i} 2 \\
\mathrm{x}_{\mathrm{i} 2}, \mathrm{y}_{\mathrm{i} 2}\end{array}$ & $\begin{array}{c}i 3 \\
x_{i 3}, y_{i 3}\end{array}$ & $\begin{array}{c}\mathrm{i} 4 \\
\mathrm{x}_{\mathrm{i} 4^{*}}, \mathrm{y}_{\mathrm{i} 4}{ }^{*}\end{array}$ & $\begin{array}{c}\text { i5 } \\
x_{i 5}, y_{i 5}\end{array}$ & $\begin{array}{c}\text { i6 } \\
x_{i 6}, y_{i 6}\end{array}$ & $\begin{array}{c}i 7 \\
x_{i 7}, y_{i 7}\end{array}$ \\
\hline
\end{tabular}

Fig.4. An Example of Mutation

In Fig. 4, for example, a mutation occurred at sensor location 4. A new location for that sensor was chosen at random. As a result, Member 1 becomes a new member of the population. The fitness of this member is then calculated in Step 2 of the USDEA.

\section{Benchmarking the USDEA}

As concluded in Section 2, the deployment strategies investigated in the literature have a different set of goals and use different metrics than the proposed USDEA, thus making a direct comparison unsuitable. Instead, a benchmarking of the USDEA was done similar to the evolutionary algorithm presented in [11]. This benchmarking has two main purposes. First, it will show that the evolutionary approach to sensor deployment employed by the USDEA will provide better sensor field fitness, and ultimately, better sensor field performance, than a brute force or a random search approach. Second, it will validate the need to use the initialization step (Step 1) of the USDEA in order to achieve high fitness values for both mesh and cluster fields.

In [11], two comparisons were made during benchmarking, one with a random search and the other, with a brute force search, in the form of a parallel hill climber (PHC) algorithm.

\subsection{Random Search}

The random search consists of executing Steps 1 and 2 of the USDEA repeatedly for some number of iterations. An iteration of the random search algorithm is called a generation. At the end of the first generation, the fitness of the fittest field in the population is recorded. After each subsequent generation, the algorithm compares the fitness of the fittest field of the current generation with the fitness of fittest field found to date. If the fitness is greater, the fitness of that sensor field becomes the new fitness of the random search.

\subsection{Parallel Hill Climber}

A standard benchmarking approach for an evolutionary algorithm is a comparison with a PHC algorithm, as it demonstrates the effectiveness of the crossover operator over taking a brute force approach (based exclusively on mutation) [12].

In a PHC algorithm, during the first generation, an initial population of sensor fields is constructed. The fitness of each sensor field is then determined using Step 2 of the USDEA. Steps 3, 4, and 5 are not used, but Step 6 is executed, where every gene in each chromosome is mutated with a mutation probability of 1. Subsequent generations consist of executing only Steps 2 and 6 . At the end of each generation, however, a copy of the fittest chromosome, which is the current local maxima, automatically survives to the next generation, so that the fitness of the algorithm never decreases.

Two PHC algorithms are considered in this work, one that constructs its initial population using Step 1 of the USDEA. The second creates its initial population using only random sensor locations, resulting in a purely random brute force search. These algorithms are referred to as "PHC Init" and "PHC No Init", respectively.

A comparison between these two $\mathrm{PHC}$ algorithms is used to validate the utility of Step 1 of the USDEA. If the fitness of the "PHC Init" is greater than "PHC No Init", it is highly likely that this is due to Step 1. The purpose of this initialization step is to create mesh or cluster fields with a required minimum cut set size or maximum number of clusters, respectively. On the other hand, not using an initialization step and creating an initial population of sensor fields using only random sensor locations will often result in a mesh or cluster field that will not meet the threshold requirements. 


\subsection{Benchmarking Parameters}

Table 1. Parameters Used in the Benchmarking Study

\begin{tabular}{|c|c|}
\hline Parameter & Value \\
\hline Size of Area & $100 \times 100$ kiloyards \\
\hline Number of Sensors & 10 \\
\hline Population Size & 22 kiloyards \\
\hline Communications Range & 11 kiloyards \\
\hline Sensing Range & 1 to 5 links \\
\hline $\begin{array}{c}\text { Minimum Cut Set Size } \\
\text { (MCSS) }\end{array}$ & 2 to 5 \\
\hline $\begin{array}{c}\text { Maximum Number of } \\
\text { Clusters (MNC) }\end{array}$ & Top 70\% of fields \\
\hline Crossover & 0.05 \\
\hline $\begin{array}{c}\text { USDEA Mutation } \\
\text { Probability }\end{array}$ & 300 \\
\hline Number of Generations & 10 runs per algorithm \\
\hline Number of Runs &
\end{tabular}

Table 1 records the parameters used for each algorithm in the benchmarking study. Note that the communications range is twice the sensing range. This is done so any sensing coverage overlaps only occur as a result of the requirements for the mesh and cluster fields, namely the topology's MCSS or MNC values. Doing so allows for a study of how effectively each algorithm can form each topology and then improve its fitness. In the subsections that follow, the fitness of mesh fields are presented as studies under Case 1 and the fitness of cluster fields as Case 2. All runs for each algorithm were done using Matlab.

\subsection{Case 1-Mesh Fields}

Mesh fields are desirable due to their link redundancy when the environment is harsh. A moderate link redundancy with an MCSS of 2 or 3 should suffice for most networks. However, this study has been extended to an MCSS of 5 to illustrate the complexity in improving fitness, and ultimately, sensing performance of the field, when imposing such a high connectivity requirement.

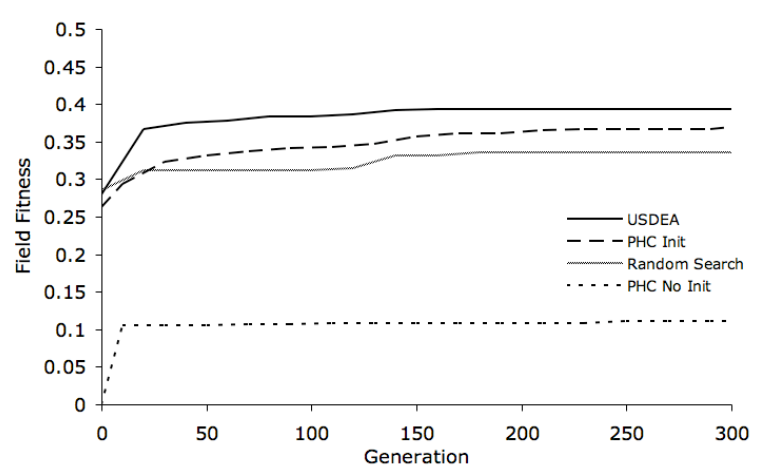

Fig.5. Benchmarking for a Mesh Field with an MCSS of 2

Fig. 5 shows the average fitness of the fittest sensor field from each generation over the 10 runs of each algorithm. In the case of mesh fields with an MCSS of 2, the PHC without an initialization step, labeled in Fig. 5 as "PHC No Init", performed quite poorly. This was a result of the algorithm using random sensor locations to construct its initial population of sensor fields. In fact, the algorithm was unable to form a single mesh field with an MCSS of 2 in 7 out of 10 runs, hence the low average fitness values. In contrast, the PHC with initialization step, labeled as "PHC Init", performed significantly better than the "PHC No Init" since it used Step 1 of the USDEA to construct its initial population of sensor fields. As a result, the "PHC Init" algorithm was able to construct many mesh fields with an MCSS of 2 , which it could then evolve using only mutation.

However, the USDEA, which uses not only a moderate amount of mutation, but also crossover, outperforms the "PHC Init" algorithm by $6.53 \%$. Since both algorithms use Step 1 of the USDEA and mutation, it can be conjectured that the use of crossover in the USDEA is quite valuable.

Lastly, the USDEA also outperforms the random search algorithm by $17.09 \%$ since the random search only creates initial populations of sensor fields using Step 1 of the USDEA, but does not perform crossover or mutation. 


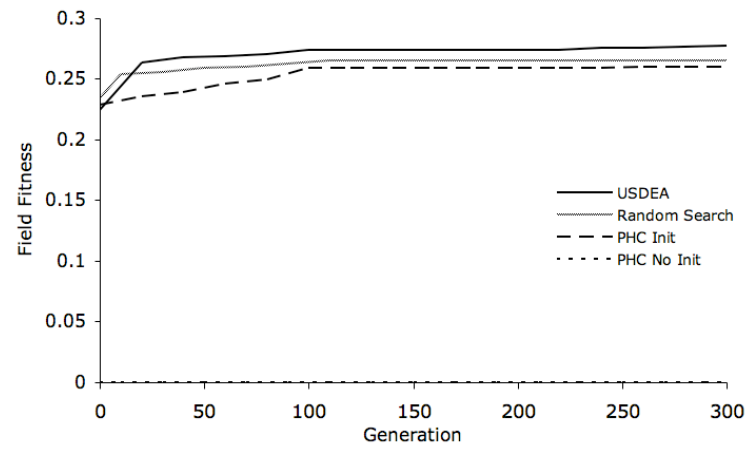

Fig.6. Benchmarking for a Mesh Field with an MCSS of 4

When the MCSS threshold is increased to 4, shown in Fig. 6, the performance improvement of the USDEA over the "PHC Init" and the random search is less. The reason for this is twofold. First, it is difficult to construct a field with an MCSS of 4 due to the strictness of the connectivity requirements. Secondly, once a field with this high link redundancy is created, adjusting the positions of sensors, while still maintaining the same connectivity, becomes quite complex. Furthermore, since sensors must be placed close together in order to meet connectivity requirements, the fitness of the sensor field decreases in comparison with mesh fields with smaller MCSS values.

It is worth noting in Fig. 6 that the "PHC No Init" never achieves a field with fitness greater than zero. This signifies that using a pure brute force approach has a high probability of failure for creating a field with an MCSS of 4. Such a finding validates Step 1 of the USDEA for mesh fields since it is able to construct many fields that meet connectivity requirements for any MCSS investigated in this section.

Table 2. USDEA Improvement Statistics for Mesh Fields

\begin{tabular}{cccc}
\hline MCSS & $\delta_{\text {PHC_init }}$ & $\delta_{\text {PHC_no_init }}$ & $\delta_{\text {Random }}$ \\
\hline 1 & $4.57 \%$ & $7.95 \%$ & $22.30 \%$ \\
2 & $6.53 \%$ & $256.07 \%$ & $17.09 \%$ \\
3 & $5.90 \%$ & $\infty$ & $8.80 \%$ \\
4 & $6.69 \%$ & $\infty$ & $4.51 \%$ \\
5 & $3.86 \%$ & $\infty$ & $2.88 \%$ \\
\hline
\end{tabular}

The fitness improvement $\delta$ achieved in using the USDEA over the PHC algorithms and the random search is defined in Eq. (10), where $a$ is the algorithm being compared with the USDEA.

$$
\delta_{a}=\left(\frac{\text { Fitness }_{U S D E A}}{\text { Fitness }_{a}}-1\right) * 100
$$

Table 2 shows the improvement in performance achieved by the USDEA over both PHC algorithms and the random search after 300 generations.

The differences in performance of these four algorithms can be logically attributed to the differences in intelligence of each algorithm. However, when the MCSS value is increased, the relative improvement achieved with USDEA over the "PHC Init" and random search decreases.

Sensor fields in the "PHC No Init" algorithm never achieved fitness greater than zero for MCSS values above 2 because a pure brute force approach lacks the intelligence to create sensor fields with such strict connectivity requirements. Even with an initialization step, the "PHC Init" has a difficult time creating mesh fields of any MCSS value above 2. Thus, expecting a purely random approach to sensor deployment, as used in the "PHC No Init" algorithm, to successfully create mesh fields of any MCSS value above 2 is unrealistic.

Convergence: Convergence and convergence times are important criteria for evaluating an evolutionary algorithm, as they measure how quickly an algorithm can reach a viable solution. The USDEA converges on average to a fitness value equal to $97.5 \%$ of the fitness value at Generation 300 after only 25 generations. Even the USDEA's fitness value after 25 generations is higher than any of the other algorithms for mesh fields. The low convergence time of the USDEA is due to the combination of using Step 1, along with both crossover and mutation. In terms of computation time, the USDEA achieved convergence in $3^{\dagger}$ minutes. (As a note, the full 300-generation run of the USDEA took 36 minutes on average to complete.)

Both PHC algorithms took an average of 12 minutes to run 300 generations, but never converged during this time, since very few mesh fields maintained their connectivity after being mutated during each generation. Even with the initialization step, the "PHC Init" could obtain very few mesh fields with fitness above zero after mutation. Whenever a field did not meet connectivity requirements, which was a common occurrence with the PHC algorithms, its fitness was not

\footnotetext{
† MacBook Pro with a $2.33 \mathrm{GHz}$ Intel Core 2 Duo processor and 2 GB of RAM was used in this work.
} 
evaluated, hence the low runtime for mesh fields using both PHC algorithms.

Runtime for the random search was 45 minutes on average. Numerous fitness calculations had to be run for this algorithm, as the algorithm consists only of doing an initialization step during each generation. This suggests that the initialization step is quite effective in producing mesh fields that meet connectivity requirements since many fields did meet connectivity requirements and thus had to have their fitness calculated.

While the importance of the initialization step and mutation is quite clear, the importance of additionally using crossover in the USDEA cannot be understated.

\subsection{Case 2 - Cluster Fields}

Cluster fields are valuable in situations where the environment is not harsh since they only require onehop cluster clients to maintain connectivity with a cluster head. As the number of clusters increases, a sensor field can attain more sensing coverage because the clusters, as well as the sensors located within those clusters, can be more spread out. Unfortunately, there is a cost implication associated with adding more clusters to a field, as each additional cluster requires an expensive cluster head to be added to the field. This study shows that creating a cluster field with an MNC of 2 is rather complex, but that deployment becomes less complex with an increased $\mathrm{MNC}$, albeit at a higher monetary cost for the field.

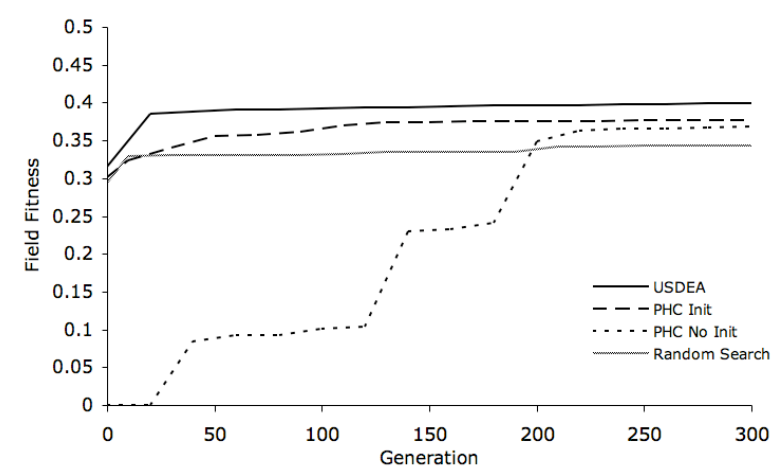

Fig.7. Benchmarking for a Cluster Field with an MNC of 2

In the case of cluster fields with an MNC of 2, shown in Fig. 7, the "PHC No Init" takes approximately 200 generations to achieve fitness similar to "PHC Init". This is because creating a field with two clusters is non- trivial since the formation of a new cluster is very sensitive to the location of the sensors in the field, as discussed in Section 3.2 When sensors are placed randomly, it is difficult to ensure that only 2 clusters are formed.

The random search achieves a low fitness even after 300 generations since it does not evolve the cluster fields once they are formed. Once again, the USDEA outperforms the other algorithms due to the combination of its initialization step, crossover, and mutation.

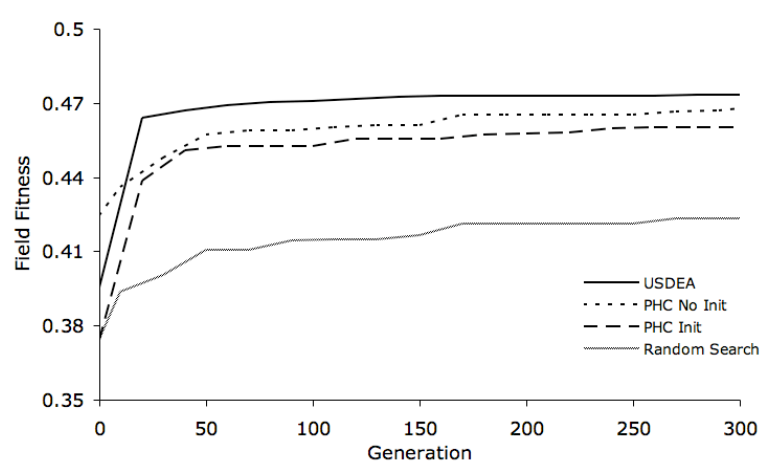

Fig.8. Benchmarking for a Cluster Field with an MNC of 5

From Fig. 8 it can be noticed that when creating a field with 5 clusters, it is slightly more beneficial for the PHC to use random sensor locations for the initial population, which is denoted by "PHC No Init" having a higher fitness than "PHC Init". It should also be noted that the fitness when using 5 clusters is higher than with 2 clusters since the clusters are more spread out and less sensing overlap occurs. Finally, Fig. 8 shows that deploying a 5-cluster field is less complex than a 2cluster field since a purely brute force approach outperformed the brute force approach using an initialization step that was used to help form clusters.

Table 3. USDEA Improvement Statistics for Cluster Fields

\begin{tabular}{cccc}
\hline MNC & $\delta_{\text {PHC_init }}$ & $\delta_{\text {PHC_no_init }}$ & $\delta_{\text {Random }}$ \\
\hline 2 & $5.94 \%$ & $8.27 \%$ & $16.26 \%$ \\
3 & $4.64 \%$ & $4.71 \%$ & $15.12 \%$ \\
4 & $3.70 \%$ & $2.66 \%$ & $12.97 \%$ \\
5 & $2.89 \%$ & $1.24 \%$ & $11.85 \%$ \\
\hline
\end{tabular}

Table 3 shows that the improvement in using the USDEA diminishes in comparison with each algorithm after each MNC increment. This finding again suggests that the deployment is easier when more clusters are 
formed since the clusters, and the sensors located within them, can be more spread out. Of course, using additional clusters comes at a cost of adding expensive cluster heads to the field.

Convergence: Similar to the mesh field benchmarking, the USDEA converges at approximately 3 minutes of runtime. Comparatively, neither PHC algorithm converges after 300 generations. Even for an MNC of 5, there remains sensitivity in the location of sensors in terms of causing additional clusters to form as a result of mutating every sensor location during each generation. Both PHC algorithms ran on average for 20 minutes, as they were able to achieve comparatively more cluster fields with the required $\mathrm{MNC}$ value than with mesh fields. Once again, the random search took on average 45 minutes to complete. Finally, the full 300 generations of the USDEA took 36 minutes to run on average.

\section{Effect of Communications Range on Field Sensing Capabilities}

Having validated the USDEA approach in the previous section, the sensing capabilities of fields generated using the USDEA is now evaluated. This evaluation is done using a Monte Carlo simulation tool called MUSICAL $^{\mathrm{TM}}$ [13]. The evaluation takes into account the impact of the communications range and the topology on the sensing capabilities of a sensor field and is the second major contribution of this article.

Two stages were involved in this study. In the first stage, for each type of sensor field, i.e. the mesh and the cluster field, the USDEA was run with the parameters provided in Table 4. The USDEA was run 100 times for each of the field types using two communications ranges, 11 and 5.5 kiloyards (kyd) and the fittest field from each run was saved.

Table 4. USDEA Parameters Used in the Communications Range and Topology Study

\begin{tabular}{|c|c|}
\hline Parameter & Value \\
\hline Size of Area & $100 \times 100$ kiloyards \\
\hline Number of Sensors & 10 \\
\hline Population Size & 100 \\
\hline Communications Range & 11 and 5.5 kiloyards \\
\hline Sensing Range & 11 kiloyards \\
\hline $\begin{array}{c}\text { Minimum Cut Set Size } \\
\text { (MCSS) }\end{array}$ & 2 to 5 \\
\hline $\begin{array}{c}\text { Maximum Number of } \\
\text { Clusters (MNC) }\end{array}$ & 25 generations \\
\hline Number of Generations & \\
\hline
\end{tabular}

\begin{tabular}{|c|c|}
\hline Crossover & Top 70\% of fields \\
\hline Mutation Probability & 0.05 \\
\hline Number of USDEA Runs & 100 per threshold value \\
\hline
\end{tabular}

These communications ranges are exactly equal to and half of, respectively, the sensing range that can be achieved by each sensor. Such communications ranges force sensing overlap between sensors and thus reduce a field's sensing capabilities as a result. In addition, connectivity restrictions placed on the sensor fields for each topology may further reduce a field's sensing capabilities.

In the second stage, the most fit sensor field found after each USDEA run was simulated 100 times, using MUSICAL $^{\mathrm{TM}}$, which evaluates the field's effectiveness in sensing underwater adversaries. Simulations in MUSICAL $^{\mathrm{TM}}$ were run for 12 hours of simulation time against 5000 randomly placed adversaries, whose course changed every hour in a random fashion. During each simulation, the number of uniquely detected adversaries was counted to determine the cumulative probability detection $C P D_{S I M}$. This value represents the expected performance of a sensor field in practice.

The results presented in this section highlight the dependence of a field's sensing capabilities on the communications range. Additionally, they also illustrate the ability of the USDEA to improve the sensing capabilities of a sensor field from the first generation to the last generation of evolution, even with strict connectivity requirements and communications ranges.

\subsection{Case 1-Mesh Fields}

In the following subsections, a series of cumulative probability of detection plots are presented. These plots were determined by running MUSICAL ${ }^{\mathrm{TM}}$ for mesh fields and cluster fields, using the two communications ranges. Included in each plot is an "Ideal" curve, which is the expected performance of a field of 10 sensors after 12 hours without any constraints on the communications range. The curve was determined analytically using Koopman's search equation [14], as discussed in [5]. In Figs. 9-12, the curves for MCSS 3 and 4 are omitted to avoid clutter, as these curves fall between the curves for MCSS 2 and 5 and do not provide any added knowledge. 


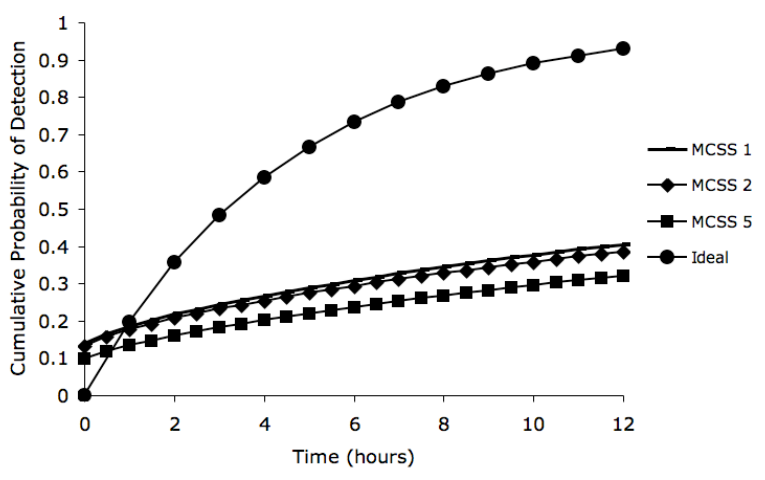

Fig.9. Average CPD of Initial USDEA Mesh Fields Communications Range $=11$ kyd

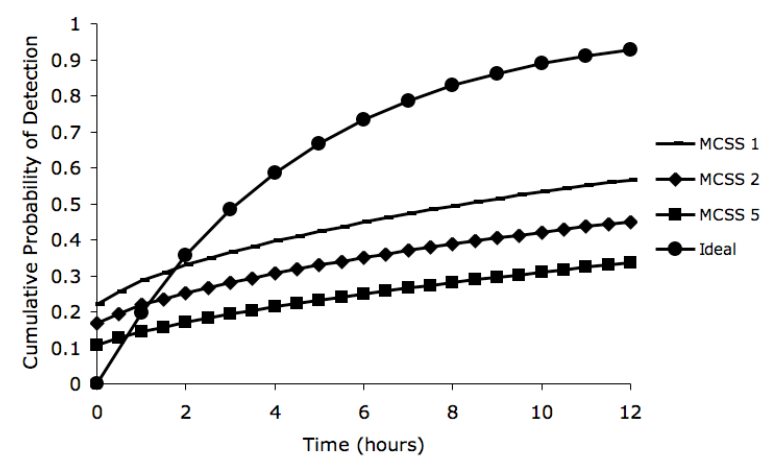

Fig.10. Average CPD of Final USDEA Mesh Fields Communications Range $=11 \mathrm{kyd}$

Figs. 9 and 10 show the average $C P D_{S I M}$ accretion over 100 simulations of the fittest sensor field from each of the 100 USDEA runs done for each MCSS. The average performance of the fittest mesh fields of the first (initial) generation is shown in Fig. 9, while Fig. 10 shows that of the most fit (best) mesh field after the $25^{\text {th }}$, and final, generation. There is a significant increase in $C P D_{S I M}$ from the initial to the final generation in all cases due to the effectiveness of the USDEA. However, there is also substantial field performance degradation as the MCSS is increased from 1 to 5 links since the sensors must be placed closer together in order to achieve higher MCSS values.

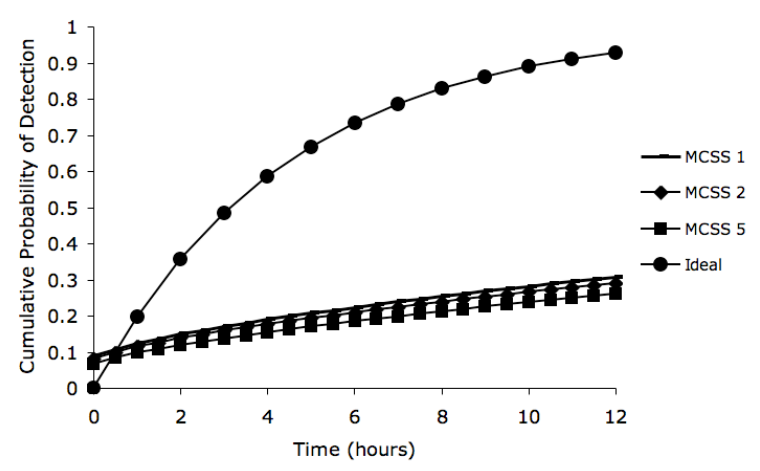

Fig. 11. Average CPD of Initial USDEA Mesh Fields Communications Range $=5.5 \mathrm{kyd}$

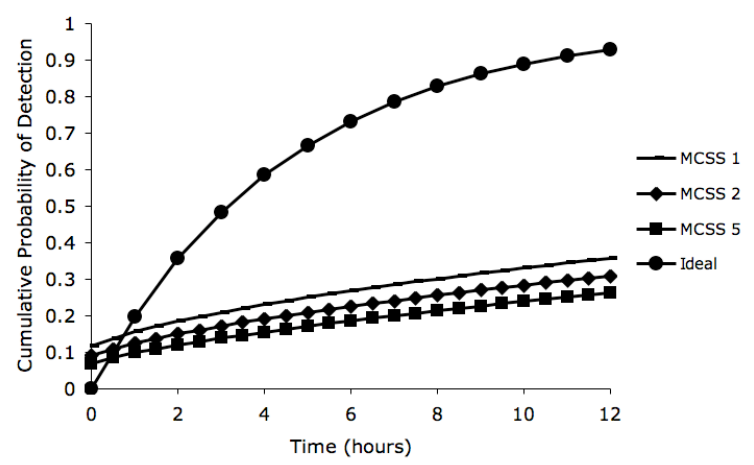

Fig.12. Average CPD of Final USDEA Mesh Fields Communications Range $=5.5 \mathrm{kyd}$

When the communications range for the sensors in a mesh field is reduced to $5.5 \mathrm{kyd}$, improvement in sensor field performance is difficult to achieve, as seen in Figs. 11 and 12. This is especially evident as the MCSS increases since both connectivity requirements and the strict communications range make optimization of the sensor locations quite complex. Also, it should be noted that the achievable field performance with a communications range of $5.5 \mathrm{kyd}$ is much less than that of $11 \mathrm{kyd}$ due to the closeness with which the sensors must be placed to provide the desired connectivity.

It should be noted that the impact of the communications range on the detection capabilities of a sensor field is much more significant than the impact of the connectivity constraints imposed by increasing the MCSS. This is intuitive since a reduction in communications range by half must result in the sensors being deployed at least twice as closely. However, increasing the MCSS does not necessarily result in such a significant change. 


\subsection{Case 2 - Cluster Fields}

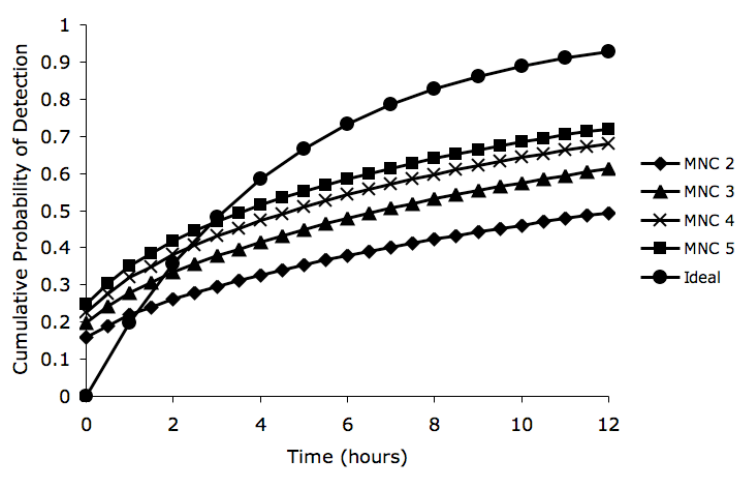

Fig.13. Average CPD of Initial USDEA Cluster Fields Communications Range $=11$ kyd

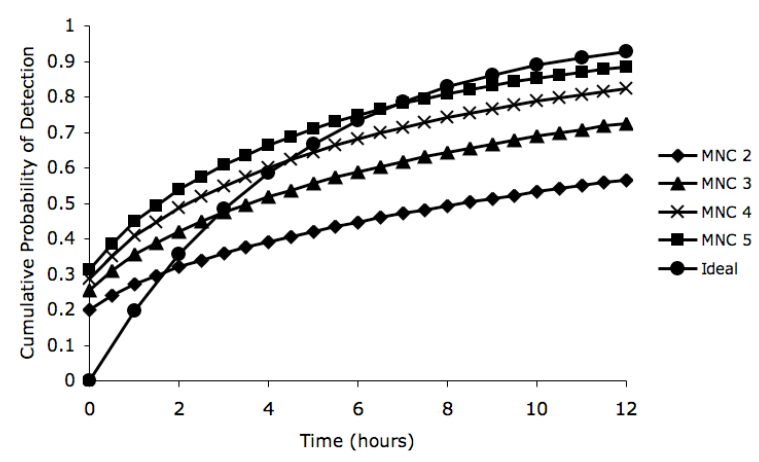

Fig.14. Average CPD of Final USDEA Cluster Fields Communications Range $=11 \mathrm{kyd}$

For cluster fields, a high level of improvement in performance can be realized for all MNC values from the initial fields, shown in Fig. 13, to the final fields, shown in Fig. 14. Less strict communications requirements afforded by the use of one-hop clusters allows for more flexibility in sensor locations during optimization of the cluster fields. As the number of clusters increases to 5 , field performance is able to reach nearly that of the Ideal case, even with a communications range equal to the sensing range. With each additional cluster added to the field (increase in $\mathrm{MNC}$ ), unique sensing coverage regions are formed and less sensing overlap occurs since fewer sensors are attempting to communicate with the cluster head.

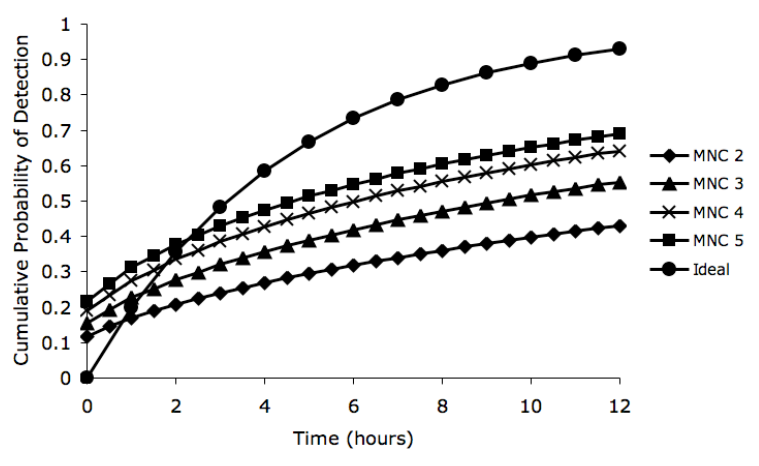

Fig.15. Average CPD of Initial USDEA Cluster Fields Communications Range $=5.5 \mathrm{kyd}$

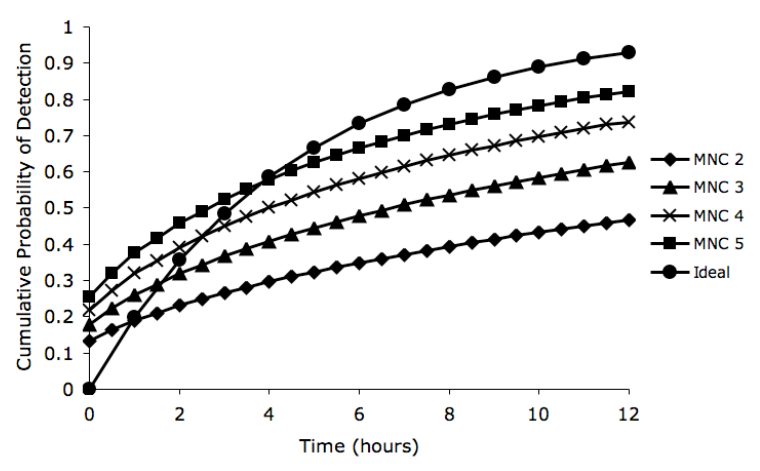

Fig.16. Average CPD of Final USDEA Cluster Fields Communications Range $=5.5 \mathrm{kyd}$

Reducing the communications range to $5.5 \mathrm{kyd}$ still results in good sensing capabilities, compared to the "Ideal" case, as seen in Figs. 15 and 16. There is degradation in performance of the cluster fields when the communications range is halved. However, the reduction in sensing capabilities for the same decrease in communications range is not as significant as seen in the mesh fields since the communications requirements for cluster fields requires cluster clients to communicate only with cluster heads. This performance improvement in sensing capabilities of the cluster fields comes at a cost of adding an expensive cluster head to the field for each additional cluster.

\section{Analysis and Discussion}

This section provides an in depth analysis of the sensor field performance results shown in the previous section. A comparison is also made between these results and the simulated sensor field detection capabilities when 
the communications range of the sensors was double the sensing range, or 22 kiloyards. It is unlikely that a communications range would be double the acoustic sensing range of a sensor due to the relatively high frequencies used for communications underwater, thus the 22 kyd sensing data is only used here for illustrative purposes.

\subsection{Case 1-Mesh Fields}

The following equations are used to calculate the metrics shown in the next two tables, as well as the first two tables in the next subsection.

$$
\begin{aligned}
\delta_{U S D E A} & =\left(\frac{\text { Fitness }_{\text {Final }}}{\text { Fitness }_{\text {Initial }}}-1\right) * 100 \\
\lambda_{\text {SIM }} & =\left(\frac{C P D_{\text {Final }}}{C P D_{\text {Initial }}}-1\right) * 100
\end{aligned}
$$

Eq. (11) shows the improvement in fitness $\delta_{U S D E A}$ from the fittest field of the first generation to the fittest field of the $25^{\text {th }}$, and final, generation for a single threshold (MCSS or MNC) value. Eq. (12) represents $\lambda_{S I M}$, the improvement in $C P D_{S I M}$ after 12 hours of simulation time using the fittest field from the first generation to the fittest field of the final generation.

$$
\begin{aligned}
\mu_{i, n} & =\left(\frac{C P D_{\gamma_{n}}}{C P D_{\gamma_{i}}}-1\right) * 100 \\
\nabla_{n, n+1} & =\left(\frac{C P D_{\gamma_{n+1}}}{C P D_{\gamma_{n}}}-1\right) * 100
\end{aligned}
$$

When a field's threshold value $\gamma$ is increased from its smallest value $i$ to any higher value $n$, there is a change in $C P D_{S I M}$, which is represented in Eq. (13). This change $\mu_{i, n}$ is always a loss in the case of mesh fields. For example, in Table 5 below, a loss in sensing capabilities of $-41.93 \%$ is seen when increasing the MCSS from 1 to 3 links.

Eq. (14), however, denotes the change in fitness when incrementing from the previous threshold value $n$ 1 to the current threshold value $n$, which is known as the marginal utility $\boldsymbol{\nabla}_{\boldsymbol{n}-\mathbf{1}, \boldsymbol{n}}$ of the increment. The marginal utility is always negative for mesh fields. For example, increasing the MCSS from 2 to 3 links results in a change of $-15.97 \%$ in $C P D_{S I M}$.

Table 5. Effect of Increasing MCSS, Communications Range 11 Kiloyards

\begin{tabular}{ccccc}
\hline MCSS & $\delta_{U S D E A}$ & $\lambda_{\text {SIM }}$ & $\mu_{1, n}$ & $\nabla_{n-1, n}$ \\
\hline 1 & $41.36 \%$ & $39.76 \%$ & - & - \\
2 & $18.63 \%$ & $16.83 \%$ & $-25.97 \%$ & $-25.97 \%$ \\
3 & $12.99 \%$ & $11.51 \%$ & $-41.93 \%$ & $-15.97 \%$ \\
4 & $8.54 \%$ & $7.69 \%$ & $-57.35 \%$ & $-15.41 \%$ \\
5 & $5.07 \%$ & $4.43 \%$ & $-69.13 \%$ & $-11.79 \%$ \\
\hline
\end{tabular}

Table 5 first shows the increase in fitness by the USDEA after 25 generations of evolution for each MCSS with a communications range of $11 \mathrm{kyd}$. It can be seen that there is a decrease in $\delta_{U S D E A}$ as the MCSS increases since the added strictness of the communications requirements causes the sensors to be deployed closer together. The $\lambda_{S I M}$ after 12 hours of simulation time also decreases as the MCSS increases for the same reasons. It is worth noting that improvements in fitness and $C P D_{S I M}$ for each MCSS closely coincide with one another. The fact that they similarly increase again validates the fitness function of the USDEA.

Additionally, it can be seen in Table 5 that as the MCSS is increased above one link, there is a significant loss in $C P D_{S I M}$ due to an increased strictness of the connectivity requirements. Also note that marginal utility actually decreases with each MCSS increment. This suggests that the impact of the MCSS on sensing capabilities decreases as the MCSS increases.

Table 6. Effect of Increasing MCSS, Communications Range 5.5 Kiloyards

\begin{tabular}{ccccc}
\hline MCSS & $\delta_{U S D E A}$ & $\lambda_{\text {SIM }}$ & $\mu_{1, n}$ & $\nabla_{\boldsymbol{n}-1, \boldsymbol{n}}$ \\
\hline 1 & $17.96 \%$ & $16.56 \%$ & - & - \\
2 & $6.97 \%$ & $6.42 \%$ & $-15.88 \%$ & $-15.88 \%$ \\
3 & $3.61 \%$ & $3.55 \%$ & $-23.79 \%$ & $-7.90 \%$ \\
4 & $2.01 \%$ & $1.94 \%$ & $-30.86 \%$ & $-7.07 \%$ \\
5 & $0.95 \%$ & $0.77 \%$ & $-36.40 \%$ & $-5.54 \%$ \\
\hline
\end{tabular}

When decreasing the communications ranges from $11 \mathrm{kyd}$ to $5.5 \mathrm{kyd}$, there is little opportunity to increase the sensing capabilities of the sensor field beyond an MCSS of 2, as seen in the first two columns of Table 6. Such a short communications range by nature causes a large amount of sensing overlap. Under strict 
connectivity requirements, it is even more complex to optimize the deployment.

Table 7. Average CPD at 12 Hours of Simulation Time for the Best Mesh Fields

\begin{tabular}{cccccc}
\hline $\begin{array}{c}\text { Comm } \\
\text { Range } \\
\text { (kyd) }\end{array}$ & MCSS 1 & MCSS 2 & MCSS 3 & MCSS 4 & MCSS 5 \\
\hline 22 & 0.8164 & 0.7030 & 0.5957 & 0.5186 & 0.4691 \\
11 & 0.5666 & 0.4498 & 0.3992 & 0.3601 & 0.3350 \\
5.5 & 0.3575 & 0.3085 & 0.2888 & 0.2732 & 0.2621 \\
\hline
\end{tabular}

Table 7 shows the average $C P D_{S I M}$ after 12 hours of simulation time for communications ranges of 22, 11 , and $5.5 \mathrm{kyd}$ for each MCSS value. There are two trends of note in this table. First, the $C P D_{S I M}$ for each communications range decreases as the MCSS increases. Secondly, the $C P D_{S I M}$ decreases for each MCSS value as the communications range decreases.

$$
\varphi_{\alpha, \beta}=\left(\frac{C P D_{\gamma_{\beta}}}{C P D_{\gamma_{\alpha}}}-1\right) * 100
$$

To quantify the second trend, let $\varphi_{\alpha, \beta}$ be the change in $C P D_{S I M}$ when the communications range of a sensor is reduced from $\alpha$ kyd to $\beta$ kyd for a threshold value $\gamma$, as given in Eq. (15). This change is always negative in the case of mesh fields, as seen in Table 8 .

Table 8. Change in Average CPD in Mesh Fields When Decreasing Communications Range

\begin{tabular}{cccc}
\hline MCSS & $\varphi_{22,11}$ & $\varphi_{22,5.5}$ & $\varphi_{11,5.5}$ \\
\hline 1 & $-44.09 \%$ & $-128.36 \%$ & $-58.49 \%$ \\
2 & $-56.29 \%$ & $-127.88 \%$ & $-45.80 \%$ \\
3 & $-49.22 \%$ & $-106.27 \%$ & $-38.23 \%$ \\
4 & $-44.02 \%$ & $-89.82 \%$ & $-31.81 \%$ \\
5 & $-40.03 \%$ & $-78.98 \%$ & $-27.81 \%$ \\
\hline
\end{tabular}

The profound effect the communications range has on the sensing coverage of a mesh field can be seen in Table 8. For all MCSS values, the change in sensing capabilities when reducing the communications range from 22 to $11 \mathrm{kyd}$ varies from $-40.03 \%$ to $-56.29 \%$. Far worse is the change when the communications range is reduced by a factor of 4 , to $5.5 \mathrm{kyd}$, which reaches upwards of $-128.36 \%$ for an MCSS of 1 . When reducing the communications range from 11 to $5.5 \mathrm{kyd}$, the loss is not as significant as in the previous case.

However, the changes when reducing the communications range from 22 to $11 \mathrm{kyd}$ and 11 to 5.5 kyd are far larger for each MCSS than any marginal utility value shown in Tables 5 and 6, respectively, when incrementing the MCSS. Only when the MCSS is increased by more than one link does such a large change occur.

\subsection{Case 2-Cluster Fields}

Table 9. Effect of Increasing MNC, Communications Range 11 Kiloyards

\begin{tabular}{ccccc}
\hline MNC & $\delta_{U S D E A}$ & $\lambda_{S I M}$ & $\boldsymbol{\mu}_{2, n}$ & $\nabla_{\boldsymbol{n}-1, \boldsymbol{n}}$ \\
\hline 2 & $17.59 \%$ & $14.58 \%$ & - & - \\
3 & $20.12 \%$ & $18.53 \%$ & $28.24 \%$ & $28.24 \%$ \\
4 & $21.24 \%$ & $20.99 \%$ & $45.56 \%$ & $17.32 \%$ \\
5 & $22.11 \%$ & $22.90 \%$ & $56.48 \%$ & $10.92 \%$ \\
\hline
\end{tabular}

In contrast with mesh fields, in the case of cluster fields, the addition of a cluster results in a gain in $C P D_{S I M}$, according to the $\mu_{2, n}$ column in Table 9. A positive marginal utility is also seen, but it decreases as the maximum number of clusters (MNC) is increased. This decrease in marginal utility represents a tradeoff between increasing sensing capabilities and the cost of adding expensive additional cluster heads. As more clusters are added, the USDEA is able to achieve higher increases in $\delta_{U S D E A}$, and as a result, $\lambda_{S I M}$, since deployment becomes relatively less complex. This is an inverse of the effect in mesh fields, where adding more link redundancy to the field (increasing the MCSS) results in smaller increases in $\delta_{U S D E A}$ and $\lambda_{\text {SIM }}$, as deployment becomes more complex.

Table 10. Effect of Increasing MNC, Communications Range 5.5 Kiloyards

\begin{tabular}{ccccc}
\hline MNC & $\boldsymbol{\delta}_{U S D E A}$ & $\lambda_{S I M}$ & $\boldsymbol{\mu}_{2, n}$ & $\boldsymbol{\nabla}_{\boldsymbol{n}-1, \boldsymbol{n}}$ \\
\hline 2 & $9.21 \%$ & $8.58 \%$ & - & - \\
3 & $12.55 \%$ & $12.82 \%$ & $33.82 \%$ & $33.82 \%$ \\
4 & $14.09 \%$ & $15.08 \%$ & $58.02 \%$ & $24.20 \%$ \\
5 & $17.29 \%$ & $19.08 \%$ & $75.88 \%$ & $17.86 \%$ \\
\hline
\end{tabular}

Similar to the results in Table 9, Table 10 shows increases in $\lambda_{S I M}$ with each additional cluster added to the field. However, these increases, and their marginal utilities, are even larger when the communications range is reduced to $5.5 \mathrm{kyd}$. This implies that adding clusters 
when the communications range is short is extremely beneficial to increasing the field's sensing capabilities. At the same time, however, the USDEA is not able to improve the $\delta_{U S D E A}$ of these fields as much as in the 11 kyd case, since the communications range is much shorter.

Table 11. Average CPD at 12 Hours of Simulation Time for the Best Cluster Fields

\begin{tabular}{ccccc}
\hline $\begin{array}{c}\text { Comm } \\
\text { Range } \\
\text { (kyd) }\end{array}$ & MNC 2 & MNC 3 & MNC 4 & MNC 5 \\
\hline 22 & 0.7434 & 0.8759 & 0.9337 & 0.9508 \\
11 & 0.5652 & 0.7248 & 0.8227 & 0.8844 \\
5.5 & 0.4669 & 0.6248 & 0.7378 & 0.8212 \\
\hline
\end{tabular}

In Table 11, as the MNC increases, there is an increase in $C P D_{S I M}$ as each additional cluster is added to the field. This is opposite to the effect seen in mesh fields (Table 7) where the $C P D_{S I M}$ decreases with an increase in MCSS. However, there is still a decrease in $C P D_{S I M}$ as the communications range decreases, in the case of cluster fields.

Table 12. Change in Average CPD in Cluster Fields When Decreasing Communications Range

\begin{tabular}{cccc}
\hline MNC & $\varphi_{22,11}$ & $\varphi_{22,5.5}$ & $\varphi_{11,5.5}$ \\
\hline 2 & $-31.53 \%$ & $-59.22 \%$ & $-21.05 \%$ \\
3 & $-20.85 \%$ & $-40.19 \%$ & $-16.01 \%$ \\
4 & $-13.49 \%$ & $-26.55 \%$ & $-11.51 \%$ \\
5 & $-7.51 \%$ & $-15.78 \%$ & $-7.70 \%$ \\
\hline
\end{tabular}

Table 12 shows that the loss in $C P D_{S I M}$ when the communications range is reduced is not nearly as significant as in the case of mesh fields, shown in Table 8. There are two main reasons for this finding. First, the connectivity requirements for a cluster topology are not as strict as mesh fields above an MCSS of 1. More importantly, cluster fields allow for a variety of sensor coverage regions that are scattered throughout an area, instead of all sensing coverage being located in a single region, as in a mesh field. This inherent flexibility in sensor deployment allows cluster fields to perform quite well, even with a short communications range.

The main drawback of a cluster field is that there exists a bottleneck at the cluster head, thus connectivity is not robust within the network. When an environment is especially harsh, a mesh field is likely to be required, even though its sensing capabilities are hindered by the connectivity requirements. A future course of study will consider the use of a meshed multihop cluster topology to determine its feasibility in terms of sensing capabilities with the added connectivity requirements to the cluster.

This analysis has shown the impact of both the communications range and a sensor network's topology on the sensing capabilities of a sensor field. No such study exists in the literature to the knowledge of the authors.

\section{Conclusions}

The Underwater Sensor Deployment Evolutionary Algorithm (USDEA) has shown the ability to create highly capable sensing fields, even with strict communications ranges and connectivity requirements. It provides underwater sensor field designers with a deployment strategy that not only takes into account six factors of the utmost importance to underwater sensor networks (sensing and communications range, sensor and deployment costs, link redundancy, range dependence, and probabilistic visitation), but also, two topologies that are well suited to the underwater environment, mesh and cluster. A benchmarking study has shown that the proposed sensor deployment strategy is superior in performance to a parallel hill climber with and without an initialization step, as well as a random search. Finally, the effects of communications range, in conjunction with various threshold values, on field sensing capabilities were studied for both mesh and cluster fields. It was determined that cluster fields are superior to mesh fields in terms of field detection capabilities when covering a large area of interest. However, mesh fields will likely be required when environmental conditions are especially harsh, in order to maintain connectivity between sensors, the surface station, and the decision makers.

\section{Acknowledgements}

The authors would like to thank Professor Sumita Mishra for her efforts in editing this article.

\section{References}

1. I.F. Akyildiz, D. Pompili, and T. Melodia. "Underwater acoustic sensor networks: research challenges", Ad Hoc Networks, Volume 3, Issue 3, May 2005, Pages 257-279. 
2. J. Partan, J. Kurose, and B.N. Levine. "A survey of practical issues in underwater networks", Proceedings of the 1st ACM international Workshop on Underwater Networks, Los Angeles, CA, USA, September 25, 2006. WUWNet '06, p. 17-24.

3. D. Pompili, T. Melodia, and I.F. Akyildiz. "Deployment analysis in underwater acoustic wireless sensor networks," Proceedings of the 1st ACM international Workshop on Underwater Networks, Los Angeles, CA, USA, September 25, 2006. WUWNet '06, p. 48-55.

4. R.J. Urick. Principles of Underwater Sound, $3^{\text {rd }}$ Edition, Peninsula Publishing, Los Altos, CA, 1983.

5. E.F. Golen, N. Shenoy, and B.I. Incze. "Underwater Sensor Field Design Using Game Theory", Military Communications Conference, October 29-31, 2007.

6. S. Meguerdichian, et al. "Coverage Problems in Wireless Ad-hoc Sensor Networks", IEEE Infocom, 2001, p. 13801387.

7. T. Clouqueur et al. "Sensor Deployment Strategy for Detection of Targets Traversing a Region", Mobile Networks and Applications 8, 2003, p. 453-461.

8. Y. Zou and K. Chakrabarty, "Sensor Deployment and Target Localization Based on Virtual Forces", IEEE INFOCOM, 2003, p. 1293-1303.

9. D.B. Jourdan and O.L. de Wek. "Layout Optimization for a Wireless Sensor Network Using a Multi-Objective Genetic Algorithm", IEEE Vehicular Technology Conference, 2004, p. 2466-2470.

10. M. Stoer and F. Wagner. "A Simple Min-Cut Algorithm", Journal of the ACM, Volume 44, Number 4, July 1997, p. 585-591.

11. S.F. Preble, H. Lipson, and M. Lipson. "Novel TwoDimensional Photonic Crystals Designed By Evolutionary Algorithms", Proceedings of the SPIE, Volume 5597, 2004, pp. 118-128.

12. M. Mitchell. "An Introduction to Genetic Algorithms", MIT Press, 1996.

13. B.I. Incze and S.B. Dasinger. "A Bayesian Method for Managing Uncertainties Relating to Distributed Multistatic Sensor Search", ICIF '06, July 2006, p. 1-7.

14. B.O. Koopman. Search and Screening: General Principles with Historical Applications. Pergamon, New York, 1980. 